life. Thus, they require a multidisciplinary approach in the recognition and treatment of psychiatric symptoms.

Keywords: Huntington's disease; Psychiatric symptoms; Depression; psychiatric comorbidity

\section{COVID-19 and related topics}

\section{EPP0273}

\section{Investigation of prospective effects of emotion-} regulation difficulties and empathic dimensions on depressive symptoms during the COVID-19 outbreak in poland

M. Gambin ${ }^{1 \star}$, M. Woźniak-Prus ${ }^{2}$, M. Sękowski ${ }^{2}$, P. Holas ${ }^{1}$, A. Wnuk ${ }^{3}$, T. Oleksy ${ }^{1}$, A. Cudo ${ }^{4}$, K. Hansen ${ }^{1}$, M. Huflejt-Łukasik ${ }^{1}$, A. Łyś $^{1}$, J. Gorgol ${ }^{1}$, K. Kubicka ${ }^{1}$, G. Kmita ${ }^{1}$ and E. Lojek $^{1}$

${ }^{1}$ Department Of Psychology, University of Warsaw, Warsaw, Poland;

${ }^{2}$ Institute Of Psychology, The Maria Grzegorzewska University, Warsaw, Poland; ${ }^{3}$ The Robert Zajonc Institute For Social Studies, University of Warsaw, Warsaw, Poland and ${ }^{4}$ Faculty Of Social Sciences, The John Paul II Catholic University of Lublin, Lublin, Poland ${ }^{\star}$ Corresponding author.

doi: $10.1192 /$ j.eurpsy.2021.683

Introduction: During the COVID-19 pandemic people experience higher levels of negative emotions, as well as face many negative and intense emotions felt by others. Thus, it is important to look for risk and protective factors that allow and help individuals to regulate these negative emotions and adapt to the hardships of the COVID19 pandemic.

Objectives: The main aims of the study were to (i) test how empathic dimensions (perspective taking, empathic concern and personal distress) and emotion regulation abilities were related to intensity of depressive symptoms during the COVID-19 lockdown in Poland, as well as to (ii) check if emotion regulation difficulties and personal distress predicted slower decrease in depressive symptoms over the two months in which the number of COVID-19 cases declined in Poland.

Methods: A total of 792 participants took part in the three-wave panel study. The sample was representative of the Polish population in terms of gender, age, and place of residence. Participants completed the following online questionnaires: The Patient Health Questionnaire-9, The Difficulties in Emotion Regulation Scale Short Form, and Brief version of the Empathic Sensitivity.

Results: Significant positive correlations were found between depressive symptoms and both personal distress and emotion regulation difficulties during the lockdown. Moreover, emotion regulation difficulties were the only significant predictor of slower decrease in depressive symptoms over time during the COVID-19 pandemic.

Conclusions: It seems that interventions focused on improvement of emotion regulation abilities could be particularly beneficial in reducing depressive symptoms during the pandemic and preventing potential negative long-term outcomes.

Keywords: emotion regulation; empathy; depressive symptoms; COVID-19 pandemic

\section{EPP0274}

\section{Illness anxiety disorder}

P. Hervias Higueras ${ }^{\star}$, S. García Jorge and J. Correas Lauffer

Psiquiatría, Hospital Universitario del Henares, Coslada, Madrid., Spain

${ }^{\star}$ Corresponding author.

doi: 10.1192/j.eurpsy.2021.684

Introduction: The diagnosis of hypochondria has disappeared in the new classification of mental illness. About $25 \%$ of patients who were diagnosed with hypochondria now fall into the category illness anxiety disorder. This disorder constitutes a new diagnostic category in DSM5 and is included within the somatic symptom and related disorders.

Objectives: We propose to carry out a bibliographic review off the new diagnostic category of illness anxiety disorder.

Methods: We present the clinical case of a 27-year-old man in the context of the Covid19 pandemic.

Results: The illness anxiety disorder is characterized by being concerned about having or acquiring a serious illness. Somatic symptoms are not present, but if they are, they are of mild intensity. The level of concern is excessive or disproportionate if there is any disease or if there is a high risk of developing it. There is a high level of health anxiety and the individual is easily alarmed by personal health status. It is a disorder that tends to be chronic and recurrent. The exact comorbidity is still unknown. However, it is important to keep in mind that hypochondria concurs with anxiety disorders and depressive disorders. Treatment is based on the cognitive restructuring of bodily symptoms. In addition, exposure therapy and acceptance and commitment therapy are also effective. Regarding pharmacological treatment, SSRIs are useful in relation with comorbidity.

Conclusions: Illness anxiety disorder is characterized by significant attention to somatic concerns in medical places, making it very useful for primary care professionals.

Keywords: illness anxiety disorder; somatic symptom and related disorders; hypochondria

\section{EPP0275}

\section{Psychological well-being and employment status during the COVID-19 pandemic}

P. Valenzuela ${ }^{1}$, C. Barrientos ${ }^{1}$, F. Molina ${ }^{1}$, D. Valdés ${ }^{1}$, I. Leniz $^{2}$, G. Reginatto ${ }^{1}$, A. Basaigoitia ${ }^{3}$, M. Solis-Soto ${ }^{1}$ and M. Burrone ${ }^{1 *}$

${ }^{1}$ Instituto De Ciencias De La Salud, Universidad de O’Higgins, Rancagua, Chile; ${ }^{2}$ Dirección De Asuntos Estudiantiles, Universidad de O'Higgins, Rancagua, Chile and ${ }^{3}$ Consulting Office, Salud Global,

Sucre, Bolivia

${ }^{\star}$ Corresponding author.

doi: 10.1192/j.eurpsy.2021.685

Introduction: Several restrictive measures have been implemented to reduced COVID- 19 impact with unknown consequences on people daily life.

Objectives: The primary objective is to asses the psychosocial impact and employment status changes since lockdown COVID19 measures in Chile.

Methods: Cross-sectional study was implemented using an anonymous and self-administered online questionnaire. Adult people were invited to participate through social networks between May to 